\title{
Discrete Markov Chain Based Spectrum Sensing for Cognitive Radio
}

\author{
Mohammadreza Amini ${ }^{1}$, Asra Mirzavandi ${ }^{2}$, Mosrafa Rezaei $^{3}$ \\ ${ }^{1,3}$ Departement of Electrical and Computer Engineering, College of Engineering, Islamic Azad University, Borujerd \\ Branch, Borujerd, Iran \\ ${ }^{2}$ Departement of Electrical and Computer Engineering, College of Engineering, Islamic Azad University, Yazd Branch, \\ Yazd, Iran
}

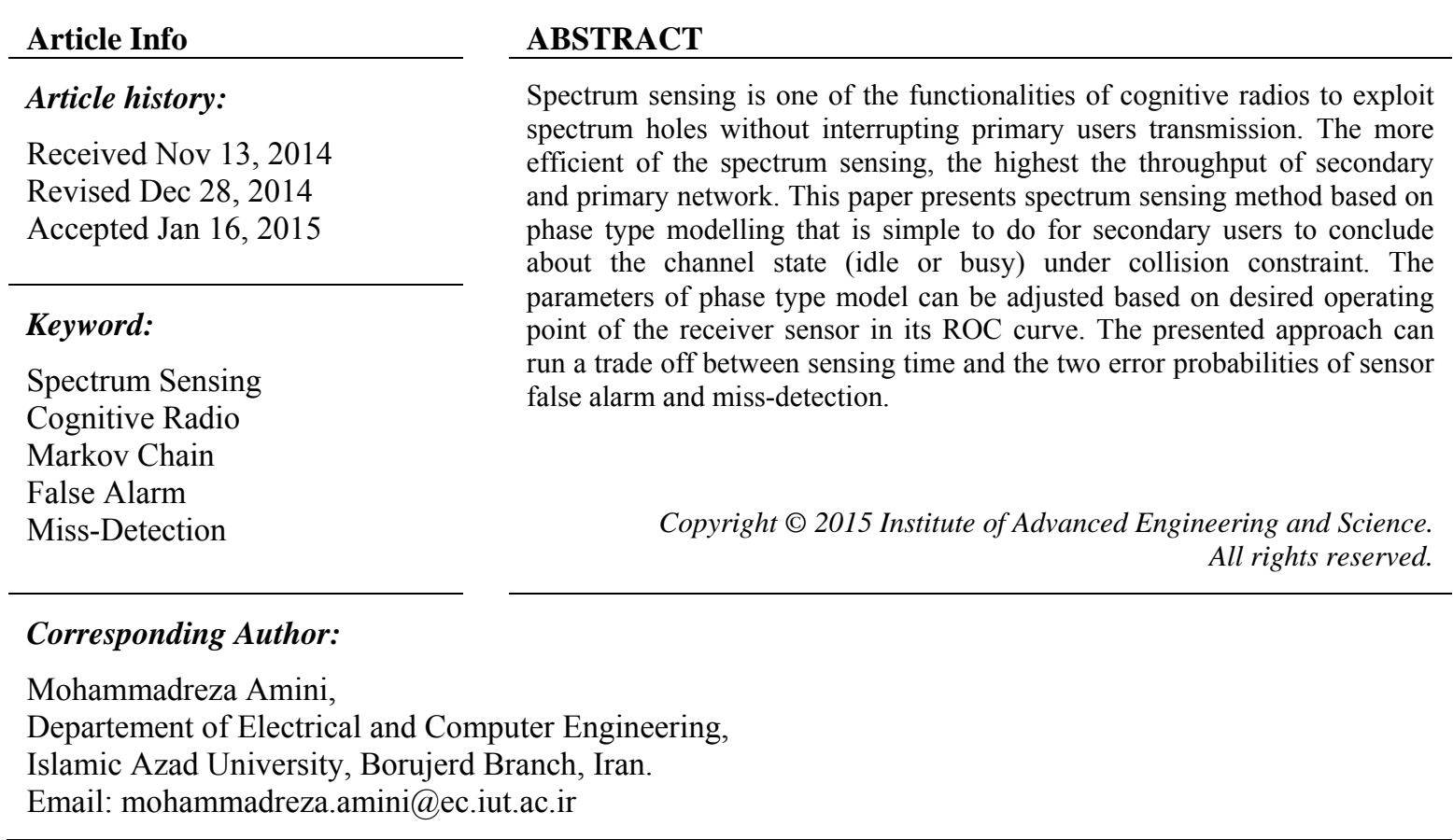

\section{INTRODUCTION}

Since spectrum in wireless communications has become valuable, cognitive radios are developed to exploit the spectrum holes in licensed bands under protective constraints for incumbent users [1, 2]. So the cognitive users (secondary users) should act intelligently in order to use the spectrum for data transmission and not to interfere with the primary users (PU) simultaneously [3, 4, 5]. In such a case, opportunistic spectrum access (OSA) named Interweave model has been evolved to enable the users dynamically access the spectrum [6, 7]. OSA has two main steps, spectrum sensing and spectrum access $[8,9]$. In the sensing step, a secondary user (SU) evaluates the spectrum bands to find idle channels and in the second step, the SU should decide on its access for data transmission $[10,11,12]$. At the end of sensing phase the SU concludes about the state of channel occupancy and there is a level of uncertainty in its decision. Some of spectrum sensing methods such as feature detection, cyclo-stationary detection and matched filter have considered physical characteristics of PU signal $[13,14,15]$ while the others exploit some general parameters of signal such as energy level along with statistical analysis to conclude about the channel occupancy [16, 17]. In this paper we propose spectrum sensing method based on phase type modelling for channel state detection. The presented approach can run a trade off between sensing time and the two error probabilities of sensor false alarm and miss-detection 


\section{SYSTEM MODEL AND PROBLEM FORMULATION}

In our proposed model, at the beginning of the sensing process the $\mathrm{SU}$ is in the zero state of Markov chain shown in figure 1 and starts to transit between states based on the probabilities depending upon received signal values sampled in each time step. The process continues till the Markov chain enters the absorb state of A. Depending on which path was traversed to enter the absorb state, the channel state is decided. With the mentioned description, the Markov chain is a discrete phase type model $\mathrm{PH}_{\mathrm{d}}(\tau, T)$ [18]. The discrete phasetype distribution is dense in the field of all discrete positive-valued distributions, that is, it can be used to approximate any discrete positive-valued distribution $[19,20]$.

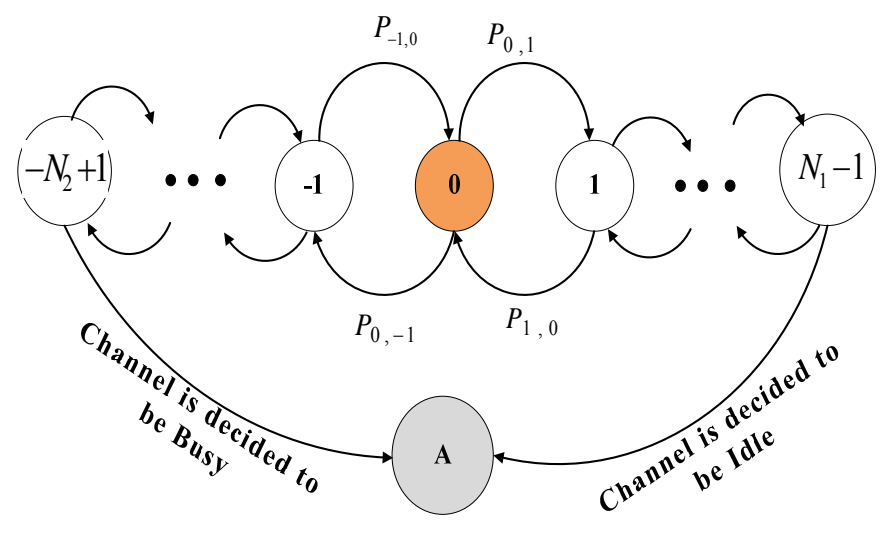

Figure 1. Phase type representation of sensing procedure

Suppose at the $k^{\text {th }}$ time step ( $k^{\text {th }}$ sample) the SU is in the $n^{\text {th }}$ state $\left(-N_{2}+1 \leq n \leq N_{1}-1\right)$. Having sampled the signal $\mathrm{r}(\mathrm{t})$, the $\mathrm{SU}$ in the chain transits to state $\mathrm{n}+1$ with probability $f_{0}\left(y_{\mathrm{k}}\right)$ and to state n-1 with probability $\left(1-f_{0}\left(y_{\mathrm{k}}\right)\right)$, in which $y_{\mathrm{k}}$ is the observation variable, see Figure 2 the observation variable is the one that a decision about channel state could be made based on such as energy level of samples. In this paper we define $y_{n} \triangleq\left|r_{n}\right|^{2}$. These transition probabilities are equal to likelihood value of signal sample due to $\mathrm{H}_{0}$ and $\mathrm{H}_{1}$ hypotheses:

$$
\begin{cases}H_{0}{ }^{\mathrm{k}}, & r(k)=\mathrm{Z}(\mathrm{k}) \\ H_{1}{ }^{\mathrm{k}}, & r(k)=\mathrm{h}(\mathrm{k}) * \mathrm{~S}(\mathrm{k})+\mathrm{Z}(\mathrm{k})\end{cases}
$$

in which $y_{\mathrm{k}}$ is the $k^{\text {th }}$ sample of signal, $\mathrm{Z}(\mathrm{k})$ is the Gaussian random variable with mean zero and some variance $\sigma^{2}$ and $\mathrm{h}(\mathrm{k})$ and $\mathrm{S}(\mathrm{k})$ are the channel coefficient and PU transmitted signal respectively. If we neglect the variation of channel coefficient during the sensing time (because the sensing phase is a short period of time) and if the samples are considered as i.i.d random variables, it can be shown that the distribution $f_{0}\left(y_{\mathrm{k}}\right)$ and $f_{1}\left(y_{\mathrm{k}}\right)$ are chi-square which are shown in equation (2) and (3) $[21,22]$.

$$
\begin{aligned}
& f_{0}=P\left(y_{n} \mid H_{0}\right)=\frac{1}{\sigma} e^{-\frac{y_{n}}{\sigma}} \mathbb{I}\left(y_{n}>0\right) \\
& f_{1}=P\left(y_{n} \mid H_{1}\right)=\frac{1}{\sigma^{2}} e^{-\frac{y_{n}+g}{\sigma^{2}}} I_{0}\left(\sqrt{\frac{g y_{n}}{\sigma} / 2}\right)
\end{aligned}
$$

in which the function $\mathbb{I}$ is :

$$
\mathbb{I}\left(y_{n}>0\right)= \begin{cases}0 & y_{n}<0 \\ 1 & y_{n}>0\end{cases}
$$

and $\mathrm{I}_{0}$ is the modified Bessel function of the first kind and $\mathrm{g}$ is the channel gain.

Because signal samples are random variables due to stochastic behaviour of channel, the Markov chain is non-homogeneous and depends on $k^{\text {th }}$ sample at $k^{\text {th }}$ time step. 


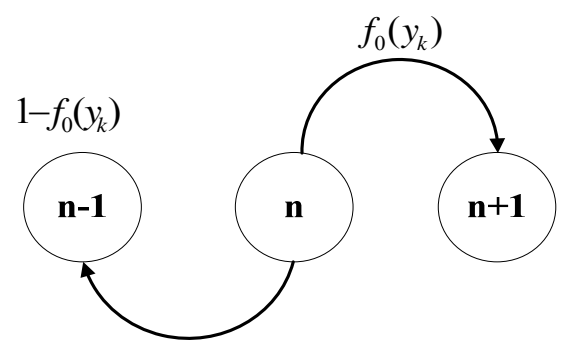

Figure 2. Transition between states for secondary user

So the equivalent phase type model (that describes the distribution of total number of samples needed to decide about channel occupancy) has the initial probability matrix $\tau$ and the transition probability matrix $T_{k}$ (at $k^{\text {th }}$ time step) described below:

$$
\begin{aligned}
& \tau=\llbracket \tau_{j} \rrbracket_{1 \times\left(N_{1}+N_{2}\right)} \\
& \tau_{j}=\left\{\begin{array}{cc}
1, j==0 \\
0, & \text { o. w }
\end{array}\right. \\
& T_{k}=\llbracket P_{i, j} \rrbracket_{\left(N_{1}+N_{2}-1\right) \times\left(N_{1}+N_{2}-1\right)}
\end{aligned}
$$

The probabilities $\mathrm{P}_{\mathrm{i}, \mathrm{j}}$ for $\mathrm{i}=-\mathrm{N}_{2}+1, \ldots, \mathrm{N}_{1}-1$ can be described as below:

$\mathrm{P}_{\mathrm{i}, \mathrm{i}+1=}=\mathrm{f}_{0}\left(\mathrm{y}_{\mathrm{k}}\right)=\mathrm{P}\left(\mathrm{y}_{\mathrm{k}} \mid \mathrm{H}_{0}\right)$

$\mathrm{P}_{\mathrm{i}-1, \mathrm{i}=1} 1-\mathrm{f}_{0}\left(\mathrm{y}_{\mathrm{k}}\right)=\mathrm{P}\left(\mathrm{y}_{\mathrm{k}} \mid \mathrm{H}_{1}\right)$

$\mathrm{P}_{\mathrm{i}, \mathrm{j}}=0$ for $\mathrm{j} \neq \mathrm{i}+1$ and $\mathrm{j} \neq \mathrm{i}-1$

Now we have to answer two questions.

1- How to compute $\mathrm{N}_{1}$ and $\mathrm{N}_{2}$ for the chain?

2- How to introduce the collision probability constraints in to the sensing scheme to protect PU signal from interference?

It is obvious that the more the value of $\mathrm{N}_{1}$, the more samples needed to decide about being idle and also the more the value of $N_{2}$, the more samples needed to decide about being busy. If both $N_{1}$ and $N_{2}$ increase the final decision has high level of certainty but the time to decision about channel occupancy grows rapidly. In other words, the values of $\mathrm{N}_{1}$ and $\mathrm{N}_{2}$ directly impact on the probability of false-alarm and missdetection. Therefore if these probabilities are known previously, the values for $\mathrm{N}_{1}$ and $\mathrm{N}_{2}$ can be calculated accordingly. To meet the collision constraint, the operating point of sensor in its ROC curve must be adjusted so that:

$$
\left\{\begin{array}{l}
P_{\text {miss-det }}=P_{\text {collision }} \\
P_{\text {fa }}=P_{\text {prescribed value }}
\end{array}\right.
$$

then the problem of finding $\mathrm{N}_{1}$ and $\mathrm{N}_{2}$ turns to calculating these probabilities. So we have:

$$
\begin{aligned}
& P_{\text {miss-det }}=\mathrm{P}\left(\mathrm{H}_{0} \mid \mathrm{H}_{1}\right)=\sum_{\mathrm{N}=\mathrm{N}_{1}}^{\infty} \mathrm{P}\left(\mathrm{H}_{0} \text { and } \mathrm{n}_{\mathrm{s}}=\mathrm{N} \mid \mathrm{H}_{1}\right) \\
& P_{f a}=\mathrm{P}\left(\mathrm{H}_{1} \mid \mathrm{H}_{0}\right)=\sum_{\mathrm{N}=\mathrm{N}_{2}}^{\infty} \mathrm{P}\left(\mathrm{H}_{1} \text { and } \mathrm{n}_{\mathrm{s}}=\mathrm{N} \mid \mathrm{H}_{0}\right)
\end{aligned}
$$

where $\mathrm{n}_{\mathrm{s}}$ is the total number of samples needed for decision. Conditioned on samples value the probability of miss-detection can be derived as: 


$$
P_{\text {miss-det }}=\sum_{\mathrm{N}=\mathrm{N}_{1}}^{\infty} \int \ldots \int \mathrm{P}\left(\mathrm{H}_{0} \text { and } \mathrm{n}_{\mathrm{s}}=\mathrm{N} \mid \mathrm{H}_{1}, \mathrm{Y}^{(\mathrm{N})}=\mathrm{y}^{(\mathrm{N})}\right) \times \mathrm{f}_{\mathrm{Y}^{(\mathrm{N})}}^{\mathrm{y}(\mathrm{N})} \mathrm{d}\left(\mathrm{y}^{(\mathrm{N})}\right)
$$

Assuming signal samples are i.i.d and considering the condition $\mathrm{H}_{1}$ it can be written as:

$$
\mathrm{f}_{\mathrm{Y}(\mathrm{N})}^{\mathrm{y}(\mathrm{N})}=\prod_{\mathrm{i}=1}^{\mathrm{N}}\left(\mathrm{f}_{1}\left(\mathrm{y}_{\mathrm{i}}\right)\right)
$$

We have also:

$$
\begin{aligned}
\mathrm{P}\left(\mathrm{H}_{0} \text { and } \mathrm{n}_{\mathrm{s}}=\mathrm{N} \mid \mathrm{H}_{1}, \mathrm{Y}^{(\mathrm{N})}=\mathrm{y}^{(\mathrm{N})}\right) & \\
& =\mathrm{P}\left(\text { SU goes from state } 0 \text { to } \mathrm{N}_{1}-1 \text { in }(\mathrm{N}-1) \text { steps }|| \mathrm{H}_{1}, \mathrm{Y}^{(\mathrm{N})}=\mathrm{y}^{(\mathrm{N})}\right) \times \mathrm{P}\left(\text { SU goes from state } \mathrm{N}_{1}\right. \\
& \left.-1 \text { to A at the final step }|| \mathrm{H}_{1}, \mathrm{Y}^{(\mathrm{N})}=\mathrm{y}^{(\mathrm{N})}\right)
\end{aligned}
$$

Proceeding to compute we have:

$P\left(\right.$ SU goes from state 0 to $N_{1}-1$ in $(N-1)$ steps ||$\left.H_{1}, Y^{(N)}=y^{(N)}\right)=\left[T_{1}, \ldots T_{N-1}\right]_{0, N_{1}-1}$

$P\left(\right.$ SU goes from state $N_{1}-1$ to state $A$ at the final step ||$\left.H_{1}, Y^{(N)}=y^{(N)}\right)=f_{0}\left(y_{N}\right)$

where $T_{i}$ is the probability matrix of phase type computed for $i^{\text {th }}$ sample and $[P]_{i, j}$ means the entry of matrix $P$ in row $\mathrm{i}$ and column $\mathrm{j}$.

$$
P_{\text {miss-det }}=\sum_{\mathrm{N}=\mathrm{N}_{1}}^{\infty} \int \ldots \int\left[\left\{\prod_{\mathrm{j}=1}^{\mathrm{N}-1} \mathrm{~T}_{\mathrm{j}}\right\}_{0, \mathrm{~N}_{1}-1} \mathrm{f}_{0}\left(\mathrm{y}_{\mathrm{N}}\right) \prod_{\mathrm{i}=1}^{\mathrm{N}}\left(\mathrm{f}_{1}\left(\mathrm{y}_{\mathrm{i}}\right)\right)\right] \mathrm{d}\left(\mathrm{y}^{(\mathrm{N})}\right)
$$

in which $\mathrm{f}_{1}\left(\mathrm{y}_{\mathrm{i}}\right)$ is the probability density function of observation variable under $\mathrm{H}_{1}$ hypothesis. The same procedure can be applied for the false-alarm probability to derive:

$$
\begin{aligned}
& P_{f a}=\mathrm{P}\left(\mathrm{H}_{1} \mid \mathrm{H}_{0}\right)=\sum_{\mathrm{N}=\mathrm{N}_{1}}^{\infty} \mathrm{P}\left(\mathrm{H}_{1} \text { and } \mathrm{n}_{\mathrm{s}}=\mathrm{N} \mid \mathrm{H}_{0}\right) \\
& \mathrm{P}_{\mathrm{fa}}=\sum_{\mathrm{N}=\mathrm{N}_{2}}^{\infty} \int \ldots \int\left[\left\{\prod_{\mathrm{j}=1}^{\mathrm{N}-1} T_{j}\right\}_{0,-N_{2}+1} \times\left(1-f_{0}\left(\mathrm{y}_{\mathrm{N}}\right)\right) \prod_{\mathrm{i}=1}^{\mathrm{N}}\left(\mathrm{f}_{0}\left(\mathrm{y}_{\mathrm{i}}\right)\right)\right] \mathrm{d}\left(\mathrm{y}^{(\mathrm{N})}\right)
\end{aligned}
$$

Considering the equations (7), (8) and (9) can be solved numerically for the minimum values of $N_{1}$ and $N_{2}$ to have the minimum sensing time. Remember that $P_{f a}$ and $P_{\text {miss-det }}$ are non-increasing function of computation of $N_{1}$ and $N_{2}$ respectively so the integrals go to zero quickly as $N_{1}$ and $N_{2}$ increase. In general the two derived probabilities are not so tractable to work with, that is because of non-homogeneous property of a Markov chain. Changing the transition law between the states results in more tractable equations which is in our next study.

\section{NUMERICAL RESULT}

In this section probability of false-alarm and miss-detection are plotted versus $\mathrm{N}_{1}$ and $\mathrm{N}_{2}$. It should be noted that probability of miss-detection and false-alarm are dependent on both $\mathrm{N}_{1}$ and $\mathrm{N}_{2}$ but it can be easily inferred that their dependencies are insignificant to $N_{2}$ and $N_{1}$ respectively. Simulation parameter and their values are shown in the Table1. Figure 3 and 4 show the two probabilities versus $N_{1}$ and $N_{2}$. To have a certain probability of false-alarm and collision, such curves can be drawn to calculate the number of states or equations (8) and (9) can be solved numerically for $\mathrm{N}_{1}$ and $\mathrm{N}_{2}$. 
Table 1. Simulation parameters

\begin{tabular}{cc}
\hline Parameter & Value \\
\hline$\sigma$ & 1 \\
$\mathrm{~g}$ & 1 \\
\hline
\end{tabular}

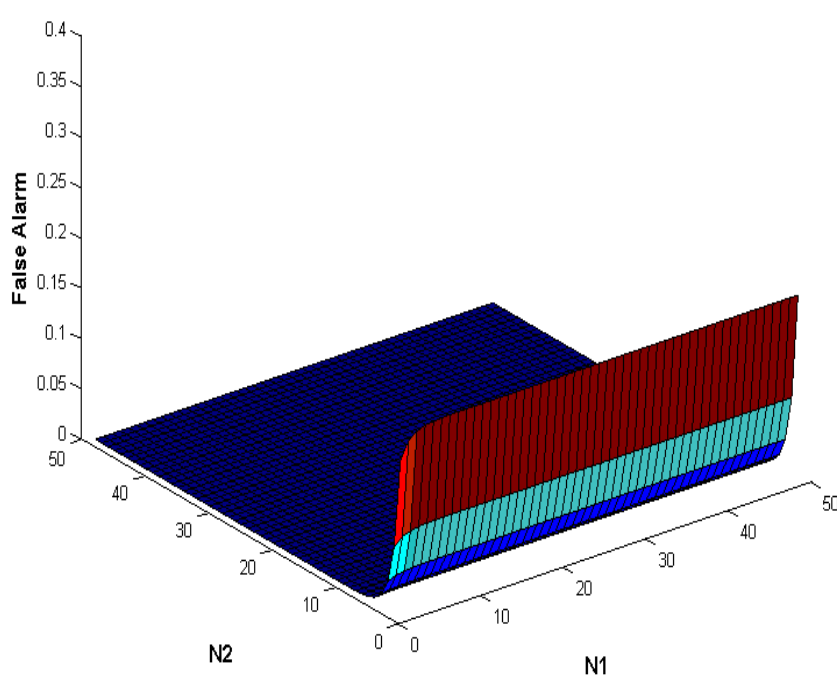

Figure 3. False alarm probability versus N1 and N2

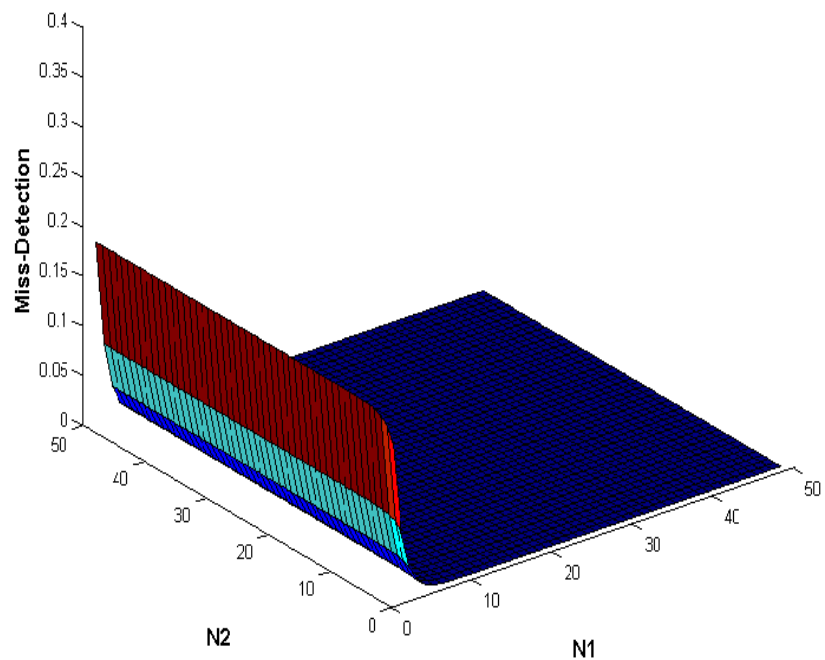

Figure 4. Miss-Detection probability versus N1 and N2

In this part, we compare our presented method with the most common spectrum sensing algorithm, i.e., energy detector. Energy detectore are known because they are simple to do for cognitive radios and do not need prior information of PU signal. Other sensing approaches are complex and have to know some prior knowledge about PU signal. The relation between the two probabilities in such a detector under the assumption of independency of primary signal and noise and the assumption of Gaussian zero mean for noise is as follows [23].

$$
P_{f a}(\tau)=Q\left(\sqrt{2 \gamma+1} Q^{-1}\left(P_{d}\right)+\sqrt{\tau f_{s}} \gamma\right)
$$


In which $\tau, \gamma, \mathrm{f}_{\mathrm{s}}$ are the sensing time, signal to noise ratio and sampling frequency respectively. $\mathrm{P}_{\mathrm{d}}$ is the probability of detedction which is the complement of $\mathrm{P}_{\text {miss-det }}$.

Equivalently, based on the number of sensing samples $(\mathrm{N})$ equation (10) will be changed to:

$$
N=\frac{1}{\gamma^{2}}\left(Q^{-1}\left(P_{f a}\right)-Q^{-1}\left(P_{d}\right) \sqrt{(2 \gamma+1)}\right)^{2}
$$

If we assume that the noise power (variance) is unity (to compare with the achieved curves in the numerical results) and under the same simulation environment, the expected number of samples needed to reach a decision about the channel state with $\mathrm{P}_{\mathrm{fa}}=0.1$ and $\mathrm{P}_{\text {miss-det }}=0.05$ is plotted in Figure 5 . As it can be seen, the number of samples to decide about the channel state is considerably lower in the presented method than to the energy detector; hence the sensing time becomes less.

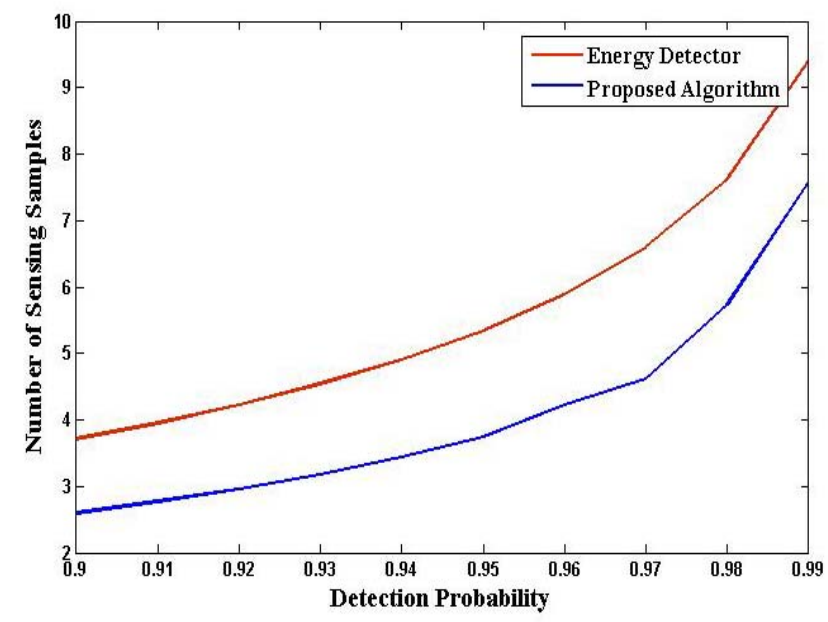

Figure 5. A decision about the channel state with $\mathrm{P}_{\mathrm{fa}}=0.1$ and $\mathrm{P}_{\text {miss-det }}=0.05$

\section{CONCLUSION}

In this paper spectrum sensing based on discerete Markov chain was presented. The presented approach is so simple to do to for secondary users to decide on channel occupancy. The parameters of the proposed model are adjusted so that the collision probability imposed by the primary network on the secondary network is met. Furthermore the presented approach gives us to adapt the sensing time. It means when the received signal power of primary user is high, the sensing time takes much less than that of for low signal power, while maintaining the operating point of the sensor constant (the false alarm and miss-detection probabilities remain unchanged). We validate our analysis by simulation and compared the proposed method with energy detector, the most common spectrum sensing algorithm.

\section{ACKNOWLEDGEMENTS}

This Study was supported by Islamic Azad University, Borujerd Branch, Iran. The authors would like to acknowledge staffs of university.

\section{REFERENCES}

[1] J. Mitola III and G. Maguire Jr. "Cognitive radio: making software radios more personal". Personal Communications, IEEE. vol. 6, no. 4, pp. 13-18, 1999.

[2] J.I. Mitola. "Cognitive Radio: An Integrated Agent Architecture for Software Defined Radio Dissertation". Dissertation Royal Institute of Technology Sweden. vol. 294, no. 3, pp. 66-73, 2000.

[3] S. Haykin. "Cognitive radio: brain-empowered wireless communications". IEEE J. Sel. Areas Commun. vol. 23, no. 2, pp. 201-220, 2005.

[4] Akyildiz, W. Lee, M. Vuran, and S. Mohanty. "Next generation/ dynamic spectrum access/cognitive radio wireless networks: A survey". Computer Networks. vol. 50, no. 13, pp. 2127-2159, Sep. 2006.

[5] M.M. Dermott. “Cognitive Sensor Platform”. Int. J. Electr. Comput. Eng. vol. 4, no. 4, pp. 520-531, Aug. 2014. 
[6] Q. Zhao and B. M. Sadler. "A Survey of Dynamic Spectrum Access”. IEEE Signal Processing Magazine. May 2007.

[7] M. Song, C. Xin, Y. Ahzo, and X. Cheng. "Dynamic Spectrum Access: From Cognitive Radio To Network Radio". IEEE Wireless Communications. February 2012

[8] Biglieri, E., Goldsmith, A.J., Greenstein, L.J., Mandayam, N., \& Poor, H.V. (2012). Principles of Cognitive Radio. Cambridge: Cambridge University Press.

[9] K. Sithamparanathan and A. Giorgetti. Cognitive Radio Techniques: Spectrum Sensing, Interference Mitigation, and Localization. Artech House. 2012.

[10] Y. Xu, A. Anpalagan, Q. Wu, L. Shen, Z. Gao, and J. Wang. "Decision-Theoretic Distributed Channel Selection for Opportunistic Spectrum Access: Strategies, Challenges and Solutions". Commun. Surv. Tutor. IEEE. vol. 15, no. 4, pp. 1689-1713, Fourth 2013.

[11] K. Ren and Q. Wang. "Opportunistic spectrum access: from stochastic channels to non-stochastic channels". Wirel. Commun. IEEE. vol. 20, no. 3, pp. 128-135, Jun. 2013.

[12] Moshe Timothy Masonta, Mjumo Mzyece. Spectrum Decision in Cognitive Radio Networks: A Survey. IEEE COMMUNICATIONS SURVEYS \& TUTORIALS. VOL. 15, NO. 3, THIRD QUARTER 2013.

[13] Md. Shamim Hossain, Md. Mahabubur Rahman, Md. Ibrahim Abdullah, and Mohammad Alamgir Hossai., "Hard Combination Data Fusion for Cooperative Spectrum Sensing in Cognitive Radio". Int. J. Elektrical Comput. Eng. vol. 2 , no. 6 , pp. 811-818.

[14] Tevfik Y"ucek and H"useyin Arslan. A Survey of Spectrum Sensing Algorithms for Cognitive Radio Applications. IEEE Communications Surveys \& Tutorials. VOL. 11, NO. 1, first quarter 2009.

[15] D.D. Ariananda, M.K. Lakshmanan, and H. Nikookar. "A survey on spectrum sensing techniques for cognitive radio". in Cognitive Radio and Advanced Spectrum Management, 2009. CogART 2009. Second International Workshop on. 2009, pp. 74-79.

[16] Q. Huang and P.J. Chung. "An F-Test Based Approach for Spectrum Sensing in Cognitive Radio". Wirel. Commun. IEEE Trans. On. vol. 12, no. 8, pp. 4072-4079, Aug. 2013.

[17] Syed Sajjad Ali and Chang Liu, Minglu Jin. "Minimum Eigenvalue Detection for Spectrum Sensing in Cognitive Radio". Int. J. Electr. Comput. Eng. vol. 4, no. 4, pp. 623-630, Aug. 2014.

[18] G. Latouche and V. Ramaswami. Introduction to Matrix Analytic Methods in Stochastic Modeling. Society for Industrial and Applied Mathematics. 1987.

[19] Aslett, L.J. MCMC for Inference on Phase-type. PHD thesis. Department of Statistics, Trinity College Dublin. 2012.

[20] A. Marshall and B. Shaw. "Computational learning of the conditional phase-type (C-Ph) distribution". Comput. Manag. Sci. vol. 11, no. 1-2, pp. 139-155, 2014.

[21] S.M. Kay. Fundamentals of Statistical Signal Processing: Detection Theory. Upper Saddle River, NJ: Prentice-Hall, 1993, vol. 2.

[22] Levy, Bernard C. Principles of Signal Detection and Parameter Estimation. Springer, 2008.

[23] Y.C. Liang, Y. Zeng, E.C.Y. Peh, and A.T. Hoang. "Sensing-Throughput Tradeoff for Cognitive Radio Networks". Wirel. Commun. IEEE Trans. On. vol. 7, no. 4, pp. 1326-1337, Apr. 2008.

\section{BIOGRAPHIES OF AUTHORS}

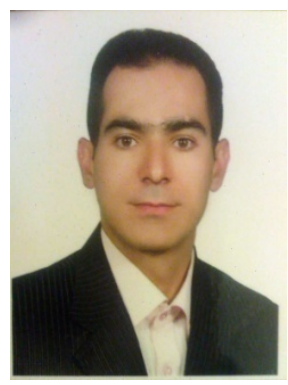

Mohammadrez Amini, is an academic member of Islamic Azad University, Borujerd Branch, Iran. He was born in 1981 and is a telecommunication researcher. He won some national awards in his research area.

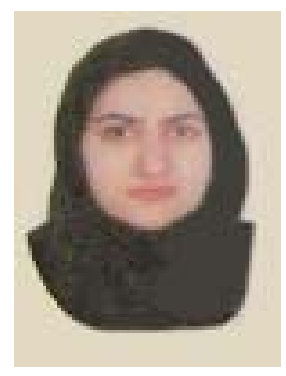

Asra Mirzavandi is now an M.sc student of electronic engineering. She was born in 1887 and is a student member of IEEE. 